\title{
A experiência compartilhada de um grupo de pesquisa da área cardiovascular no contexto da pandemia da COVID-19
}

The shared experience of a cardiovascular research group in the context of the COVID-19 pandemic

La experiencia compartida de un grupo de investigación cardiovascular en el contexto de la pandemia COVID-19

Ana Carolina Eiris Pimentel

ORCID: https://orcid.org/0000-0003-3631-010X Universidade Federal Fluminense, Brasil E-mail: anacep@id.uff.br

Amanda Cabral

ORCID: https://orcid.org/0000-0002-5707-7653 Universidade Federal Fluminense, Brasil

E-mail: amanda_cabral@id.uff.br

Fernanda Ávila da Costa Pereira

ORCID: https://orcid.org/0000-0001-8755-1371

Universidade Federal Fluminense, Brasil

E-mail: feavila@id.uff.br

João Victor Jaegger de França

ORCID: https://orcid.org/0000-0002-4720-3281 Universidade Federal Fluminense, Brasil E-mail: jaeggerjoao@id.uff.br

Kalliza Kary Rodrigues da Costa ORCID: https://orcid.org/0000-0002-6399-5995 Universidade Federal Fluminense, Brasil

E-mail:kallizarodrigues@id.uff.br

Luanna Barci Dutra da Costa

ORCID: https://orcid.org/0000-0003-0296-8667 Universidade Federal Fluminense, Brasil E-mail: luannabarci@id.uff.br

Mariany Lima Barreto de Oliveira ORCID: https://orcid.org/0000-0002-8965-6561 Universidade Federal Fluminense, Brasil E-mail: marianylbo@id.uff.br

Nathália Machado de Souza

ORCID: https://orcid.org/0000-0002-3567-0712

Universidade Federal Fluminense, Brasil E-mail: nathaliams@id.uff.br

Nathália Salazar Coelho Calegario

ORCID: https://orcid.org/0000-0002-9769-5886

Universidade Federal Fluminense, Brasil

E-mail: salazar_calegario@id.uff.br

Raquel Ravoni dos Santos

ORCID: https://orcid.org/0000-0002-8813-7954

Universidade Federal Fluminense, Brasil E-mail: raquelravoni@id.uff.br

Raquel Sangy da Costa Guimarães ORCID: https://orcid.org/0000-0003-2002-0239

Universidade Federal Fluminense, Brasil E-mail: raquelsangy@id.uff.br 


\author{
Roberta Pinto Santos \\ ORCID: https://orcid.org/0000-0002-1139-3298 \\ Universidade Federal Fluminense, Brasil \\ E-mail: roberta_santos@id.uff.br \\ Valeriana Cantanhede Rodrigues \\ ORCID: https://orcid.org/0000-0002-4921-2675 \\ Universidade Federal Fluminense, Brasil \\ E-mail: valerianarodrigues@id.uff.br \\ Yuri Pereira Gomes \\ ORCID: https://orcid.org/0000-0002-1603-7153 \\ Universidade Federal Fluminense, Brasil \\ E-mail: yuripg @id.uff.br
}

\begin{abstract}
Resumo
Objetivo: relatar a experiência dos membros de um grupo de pesquisa sobre as atividades científicas referentes à hipertensão arterial no contexto de saúde e da pandemia da Covid-19. Metodologia: estudo tipo relato de experiência acerca do período de março de 2020 a abril de 2021, a qual foi compartilhada no ambiente virtual devido ao isolamento e distanciamento social, impostos pela pandemia da COVID-19. Resultados: identificou-se de modo quantitativo, as sessões científicas, lives, posts de divulgação de eventos, participação em projeto de extensão e produção científica; enquanto no qualitativo, por meio de autorrelatos, um olhar enriquecido pela troca de impressões e valorização do aprendizado e conhecimento de todos. Conclusão: a experiência dos últimos 12(doze) meses, demostrou o quanto se faz importante a dinâmica da continuidade na discussão, participação e contribuição nas múltiplas atividades de um grupo de pesquisa.
\end{abstract}

Palavras-chave: Enfermagem; Hipertensão; Coronavírus.

\begin{abstract}
Objective: to report the experience of the members of a research group on the scientific activities related to arterial hypertension in the context of the health and pandemic of Covid-19. Methodology: an experience report study about the period from March 2020 to April 2021, which was shared in the virtual environment due to the isolation and social distance imposed by the pandemic of COVID-19. Results: scientific sessions, lives, posts to publicize events, participation in an extension project and scientific production were identified in a quantitative way; while qualitative, through self-reports, a look enriched by the exchange of impressions and appreciation of everyone's learning and knowledge. Conclusion: the experience of the last 12 (twelve) months, showed how important the dynamics of continuity in the discussion, participation and contribution in the multiple activities of a research group is important.

Keywords: Nursing; Hypertension; Coronavirus.
\end{abstract}

\begin{abstract}
Resumen
Objetivo: reportar la experiencia de los miembros de un grupo de investigación sobre las actividades científicas relacionadas con la hipertensión arterial en el contexto de la salud y pandemia de Covid-19. Metodología: estudio de relato de experiencia sobre el período de marzo de 2020 a abril de 2021, que se compartió en el entorno virtual debido al aislamiento y distancia social que impuso la pandemia de COVID-19. Resultados: se identificaron de manera cuantitativa sesiones científicas, en vivo, posts para dar a conocer eventos, participación en un proyecto de extensión y producción científica; mientras que cualitativo, a través de autoinformes, una mirada enriquecida por el intercambio de impresiones y la apreciación de los aprendizajes y conocimientos de todos. Conclusión: la experiencia de los últimos 12 (doce) meses, mostró cuán importante es la dinámica de continuidad en la discusión, participación y aporte en las múltiples actividades de un grupo de investigación.
\end{abstract}

Palabras clave: Enfermería; Hipertensión; Coronavirus.

\title{
1. Introdução
}

Em consonância com o processo educativo universitário público, o qual perpassa por ensino, pesquisa e extensão, no último ano (2020) dado a pandemia da Covid-19, e a necessidade de isolamento e do distanciamento social, várias foram as discussões nas instituições para uma adaptação exitosa, principalmente do ensino para modalidade remota. Enquanto, a extensão e a pesquisa, de igual importância na formação e contribuição para a ciência, tenham caminhado de modo específico nos diferentes espaços acadêmicos.

Nesse sentido, há relevância nos grupos de pesquisas, pois nesse espaço de relações interpessoais e interdisciplinares, subsidia-se principalmente discussões e proposições de estudos científicos. Segundo Rossiti (2018), 
"na perspectiva de "grupos", entende-se que o trabalho coletivo deriva da união, em um mesmo espaço físico ou virtual, de diferentes pessoas com interesses comuns. A oportunidade da convivência, de estar junto, aprender junto e de fazer junto, da aprendizagem compartilhada, do conhecimento de uns com os outros, das interações e das intenções de cada integrante do grupo, quando liderada com princípios norteadores e ancorada em conhecimento científico sólido, tem o potencial de se transformar em um espaço de desenvolvimento pessoal e profissional".

Portanto, o Grupo de Enfermagem de Pesquisa em Hipertensão Arterial Sistêmica (GEpHAS) de uma universidade pública federal localizada no Estado do Rio de Janeiro, sob a liderança de uma docente de enfermagem, desde março de 2020 retornou as atividades semanais para o ambiente virtual, junto a plataforma Google Meet, adotada pela universidade.

Resumidamente, a pandemia da Covid-19, foi identificada em dezembro de 2019, na cidade de Wuhan (China), onde surgiram diversos casos de pneumonia de etiologia até então desconhecida, e em janeiro de 2020, identificou-se o novo coronavírus Sars-Cov-2 por meio de uma amostra de swab de orofaringe de um dos pacientes com esse quadro (Harapan et al., 2020). Desde então, por se tratar de um vírus de rápida disseminação, a cadeia de transmissão estendeu-se até atingir picos alarmantes em múltiplos países, o que culminou num cenário pandêmico ao longo do tempo. No Brasil, o primeiro diagnóstico da Covid-19 ocorreu em 26 de fevereiro de 2020, e logo no mês seguinte foram confirmados mais casos por transmissão local (Teich et al., 2020). No país, cuja população é de 210.147.125 milhões, aproximadamente após um ano, no dia 21/04/2021, haviam sido registrados: 12.646.321 milhões de casos recuperados, 381.475 mil óbitos, e 33.807.132 de doses aplicadas (Brasil, 2021).

Portanto, no transcorrer da pandemia, tornou-se fundamental a realização de mudanças e adaptações ao quadro que se instalava, e se aplicou no atendimento a saúde aos diversos públicos. O processo de telemedicina, o qual contempla teleorientação, telemonitoramento e teleconsulta passou a ser empregado de forma pertinente e, deste período adiante, irreversível e complementar. $\mathrm{O}$ acompanhamento à distância dos pacientes através de ferramentas virtuais durante a pandemia abriu espaço para sua implementação não apenas temporária, por ter facilitado a realização de ajustes terapêuticos e a troca de informações sem a necessidade presencial imediata (Barroso et al., 2020).

Dentre as doenças cardiovasculares, a hipertensão arterial está entre as principais comorbidades associadas com risco aumentado de manifestações graves e piores prognósticos da síndrome respiratória aguda grave e lesão pulmonar causada pela infecção por Covid-19 (Rodgers \& Gibbons, 2020).

Porém, a relação entre indivíduos hipertensos e a Covid-19 no sistema respiratório não é totalmente compreendida (Barros et al., 2020). Entretanto, Cook (2020) aponta que há uma compressão sustentada que casos graves de indivíduos com Covid-19 estão relacionados a eventos vasculares importantes como manifestações micro e macro trombóticos, insuficiência renal e cardíaca.

A associação com a hipertensão arterial e seu potencial de lesão microvascular pode ser observada em casos de indivíduos que evoluem para as formas mais graves da Covid-19. A literatura evidencia que essa relação entre indivíduos hipertensos e o novo coronavírus poderia estar associada ao tratamento com medicamentos anti-hipertensivos específicos, como os inibidores da enzima de conversão da angiotensina (IECA) e bloqueadores dos receptores da angiotensina (BRA). Lembrando que a enzima conversora de angiotensina 2 (ACE-2) é a porta de entrada para o vírus SARS-Cov-2 para ter acesso às células humanas (Barros et al., 2020). A ACE-2 é amplamente expressa em pneumócitos do tipo II, coração e vasos sanguíneos, o que poderia justificar a atração do vírus pelo pulmão e sistema cardiovascular (Salazar et al., 2020). Em indivíduos hipertensos, as concentrações de ACE2 encontram-se elevadas, sendo que estes níveis aumentam ainda mais por ações de algumas drogas específicas, como os IECA e BRA (Barros et al., 2020).

No entanto, não há até o momento evidências científicas associadas à Covid-19 que sustentem a descontinuação ou cessação de medicamentos do tipo IECA ou BRA em indivíduos hipertensos (Salazar et al., 2020). O tratamento medicamentoso de indivíduos hipertensos deve ser mantido de acordo com as diretrizes atuais e orientações dos profissionais 
de saúde, independente do estágio de infecção do indivíduo pelo vírus SARS-Cov-2. A descontinuidade do tratamento pode gerar alterações dos níveis pressóricos em hipertensos, culminando em uma rápida deterioração da doença e evolução para insuficiência cardíaca, infarto agudo do miocárdio, nefropatias e outras complicações (Salazar et al., 2020).

Logo, este artigo tem por objetivo relatar a experiência de integrantes de um grupo de pesquisa sobre as atividades científicas referentes a hipertensão arterial no contexto de saúde e da pandemia da Covid-19.

\section{Metodologia}

Trata-se de um relato de experiência (Pereira et al., 2018), composto por ações quantitativas, referente ao período de março de 2020 a abril de 2021 direcionadas ao planejamento anual de atividades do grupo (sessão científica semanal, lives, minicurso, participação em evento/produção científica), e autorrelatos dos membros do Grupo de Enfermagem e Pesquisa em Hipertensão Arterial Sistêmica da Universidade Federal Fluminense (GEpHAS_UFF).

A infraestrutura de comunicação entre os membros do grupo permaneceu no aplicativo de mensagens WhatsApp já criado em 2017 e pela visibilidade em mídia social (@gephas_uff). Além disso, foram estabelecidas 2(duas) salas virtuais na Plataforma Google Meet da instituição pela responsável do grupo, respectivamente para as sessões semanais às quintas-feiras e no formato de sala de aula virtual Classroom para disposição de material para leitura e demais troca de informações.

Cabe destacar, que o grupo está registrado no Diretório de Grupos do Conselho Nacional de Desenvolvimento Científico e Tecnológico (CNPq) e tem em sua composição atual, além da líder, Doutora e Docente, 05(cinco) Enfermeiras, sendo 2(duas) Mestres, 02(duas) Mestrandas e 01(uma) Doutoranda; e 12(doze) graduandos de enfermagem.

O atendimento aos preceitos éticos para o desenvolvimento deste relato deu-se a partir da Resolução 510/2016 do Conselho Nacional de Ética em Pesquisa (CONEP), onde "não serão registradas nem avaliadas pelo sistema CEP/CONEP", dentre outros motivos "atividade realizada com o intuito exclusivamente de educação, ensino ou treinamento sem finalidade de pesquisa científica, de alunos de graduação, de curso técnico, ou de profissionais em especialização" (Inciso VIII).

\section{Resultados e Discussão}

O planejamento das atividades do grupo durante a pandemia foi interativa, sendo estabelecidas para as sessões científicas semanais, as datas, o horário e a temática. Quanto ao Minicurso GEpHAS Anual, que tem por finalidade promover a atualização sobre hipertensão arterial, direcionado a graduandos da área de saúde, o período foi mantido para o mês de julho, nos dias 27 e 28 de 2020, constituído nas modalidades síncrona e assíncrona. Ainda, estabeleceu-se 08(oito) Lives no segundo semestre de 2020 com a liderança do grupo e convidados externos de áreas interdisciplinares abordando a associação da hipertensão arterial com temáticas relevantes. Além disso, os membros do grupo foram convidados a participarem de modo voluntário por meio de contato telefônico, da etapa de teleorientação de um projeto de extensão direcionado a hipertensos resistentes, sob atendimento ambulatorial especializado do hospital universitário.

Para melhor apresentação, optou-se por disponibilizar a seguir nos Quadros 1 e 2, as atividades realizadas segundo o planejamento anual. 
Quadro 1. Distribuição quantitativa referente às atividades de sessão científica semanal, live, minicurso anual e participação na teleorientação e telemonitoramento. Niterói,2021.

\begin{tabular}{|l|c|}
\hline \multicolumn{1}{|c|}{ Atividade } & Quantitativo (em dias) \\
\hline Sessão Científica Semanal & 30 \\
\hline Live rede social Instagram & 08 \\
\hline Minicurso GEpHAS Anual & 02 \\
\hline Teleorientação/Telemonitoramento & 50 \\
\hline
\end{tabular}

Fonte: Dados GEpHAS.

Observa-se o intenso calendário proposto e que foi desenvolvido em ambiente virtual, mobilizando a contribuição e participação voluntária de cada um dos membros do grupo de pesquisa e resultando em um ótimo trabalho em grupo, dado a sequência e o quantitativo, e ainda avaliações positivas registradas pelos participantes do Minicurso e das Lives, respectivamente em formulário eletrônico (Google Forms) e no Chat.

Quanto à produção científica que tenha reunido mais de 06 participantes do grupo, identificou-se um resumo simples, um artigo científico completo (Quadro 2). Com o intuito de promover a atualização permanente dos membros e seguidores da página do Instagram (@gephas_uff) quanto a temas em geral da área cardiovascular, COVID-19, métodos de pesquisa científica e cursos gratuitos da referida área, foi realizado como atividade de divulgação permanente de eventos científicos, oriundos da Sociedade de Cardiologia do Estado do Rio de Janeiro (SOCERJ), Sociedade de Cardiologia do Estado de São Paulo (SOCESP), Sociedade de Terapia Intensiva do Estado do Rio de Janeiro (SOTIERJ), Sociedade Brasileira de Cardiologia (SBC), Portal de Periódicos da Coordenação de Aperfeiçoamento de Pessoal de Nível Superior (CAPES), Cursos do Conselho Federal de Enfermagem (COFEN) e do Instituto de Educação e Pesquisa do Hospital Moinhos de Vento.

Quadro 2. Distribuição quantitativa referente a produção científica e divulgação de eventos científicos. Niterói,2021.

\begin{tabular}{|l|ll|}
\hline \multicolumn{1}{|c|}{ Atividade } & \multicolumn{1}{c|}{ Quantitativo } \\
\hline Produção Científica & $\bullet$ 01 Resumo Publicado (Congresso RedeUnida) \\
& $\bullet$ 01 Trabalho Apresentado (Congresso RedeUnida) \\
& $\bullet$ 01 Artigo Científico (Revista Enfermagem em Foco) \\
\hline $\begin{array}{l}\text { Divulgação de Eventos Científicos } \\
\text { no Instagram }\end{array}$ & $\bullet$ 35 posts na página do Instagram \\
\hline
\end{tabular}

Fonte: Dados GEpHAS.

Parte da experiência, no formato de autorrelato, foi proposta aos membros a partir da pergunta " $O$ que representou para você membro GEpHAS a sua participação em cada uma destas atividades (Sessão Científica, Live, Artigos, Apresentação de Trabalhos e Teleorientação?)". Além disso, "na sua opinião qual foi a contribuição na sua formação?”. Portanto, nos Quadros 3 e 4, os principais pontos destacados e identificados desse conjunto de autorrelatos, respectivamente como Desafio, Aprendizado e Formação e Participação no Telemonitoramento.

Inicialmente, sobre o "Desafio", foi evidenciado pela a Lei no 14.040/2020 do Governo Federal, a aprovação em caráter excepcional, do Ensino Remoto devido ao atual contexto da pandemia da COVID-19. E na experiência do grupo, muito foi o desafio de adaptação. Pois, essa nova realidade na educação trouxe e ainda traz diversos desafios para os professores e alunos de todos os segmentos, de modo que houve necessidade de readaptação, em pouco tempo, ao uso de ferramentas digitais que até então eram pouco utilizadas. Essa rápida adaptação proporcionou diversas dúvidas e anseios sobre como manter a interação e produzir conhecimento e informação que seja multilateral e produtivo para todos (Barreto \& Rocha, 2020; Lei n. 14.040, 2020). 
Entretanto, apesar da distância física, a tecnologia abriu espaço para uma nova oportunidade de interação de docentes e discentes, os quais estabeleceram um aprendizado mútuo. E ao se verificar estudos que tratam de grupos do ensino superior e suas vivências ao longo das adversidades causadas pela pandemia, percebeu-se que o que fortaleceu seus encontros, foi principalmente a dúvida de como se estudar ou ensinar (Oliveira, 2021).

E cabe citar que encontrar o equilíbrio entre o excesso e uma a produtividade com qualidade, faz-se um grande desafio (Barreto \& Rocha, 2020).

Além disso, a participação efetiva e produtiva nos encontros remotos caminha em paralelo às barreiras relacionadas à rede de internet enfrentadas por docentes e discentes, uma vez que se enfrenta a acessibilidade e qualidade da conexão, que foram evidenciados como necessidade de evolução no país durante a pandemia (Souza, 2020).

Quadro 3. Pontos em destaque para o autorrelato dos membros do grupo de pesquisa quanto ao desafio e aprendizado. Niterói,2021.

\section{DESAFIO}

- “...o cenário mundial era de imensa incerteza, e as modificações das estratégias de ensino, pesquisa e extensão foram necessárias no meio acadêmico. Porém, mesmo em meio a desafios e adversidades, foi possível ter uma experiência única de aprendizado...".

- “...Impressionante como podemos estar unidos mesmo fisicamente distantes. Dessa forma, o GEpHAS em 2020 mostrou que é importante estarmos sempre olhando para frente e focando naquilo que podemos sempre melhorar: Conhecimento. E não foco apenas no conhecimento científico, mas no conhecimento humano, das relações interpessoais e conosco buscando sempre as nossas melhores versões, pessoais e profissionais, através do trabalho em equipe..."

- “...A iniciativa de todos os membros e da líder deste grupo de pesquisa para garantir a continuidade das atividades de forma segura em meio ao contexto pandêmico impulsionou a criação de novos caminhos para continuidade de partilhas de conhecimento, trocas de experiências e produções cientificas..."

- “...As atividades desenvolvidas no GEpHAS foram experiências pioneiras para mim, o que trouxe diversas nuances de conhecimento e aprendizagem, onde o uso das tecnologias para finalidades educacionais em favor da ciência e pesquisa, se descortina como um facilitador a ser cada vez melhor explorado..."

- “...No sentido das atividades acadêmicas, com incertezas de retorno às aulas presenciais, foi possível por meio da participação ativa no GEPHAS ter uma esperança de produtividade, orientações e trocas de experiências acadêmicas mesmo que de forma remota..."

- “...A experiência no grupo nos permitiu vencer as dificuldades vivenciadas e produzir conhecimento através de estratégias possiveis diante de um momento atípico...”

- “...Ao longo do ano de 2020, a participação em diversos eventos promovidos pelo GEpHAS, como o curso de inverno, lives ocorridas na plataforma virtual Instagram e sessões científicas, foram, indubitavelmente, experiências singulares que agregaram ricamente esta jornada como uma acadêmica de enfermagem. Mesmo durante um cenário de pandemia, foi possível adaptar-se às novas condições e meios de aprendizado proporcionadas pelo grupo de forma indireta ou direta..."

- “...Como membro do Gephas, participar de todos esses eventos foi muito gratificante, principalmente neste ano de 2020 que foi marcado pela dificuldade em todos os aspectos, pela pandemia, lutos e além disso a dificuldade do ensino remoto na faculdade, com isso, o Gephas se tornou uma válvula, uma forma de me manter com ânimo e empenhado nos estudos, principalmente por abordar temas do meu interesse..."

\section{APRENDIZADO}

- “...foram momentos de enriquecimento cientifico..."

- “...Em meio a um ano turbulento que trouxe a incerteza do ensino remoto, conheci o grupo GEpHAS. Como acadêmica, senti a necessidade de participar das atividades propostas para ampliar meu conhecimento, e a partir disso, começar a definir quais temas me trariam maior afinidade. Posso dizer que o conteúdo da Sessão Científica semanal com discussão de artigos era sempre muito pertinente, trazendo o pensamento crítico-reflexivo e promovendo o esclarecimento de dúvidas..."

- “...Certamente foi de grande aprendizado, pois exigiu compromisso e a busca por novas leituras para acrescentar durante as reuniões..."

- “...Com recursos e redes sociais, o grupo inovou ao realizar encontros virtuais, as conhecidas lives, com profissionais renomados em temáticas estabelecidas previamente para discussões. Com a referida experiência foi possível a divulgação e partilha de informações a vários profissionais da área da saúde, estudantes, docentes e pesquisadores. O resultado foi uma 
experiência extremamente rica e valiosa..."

- “...Posso dizer que mesmo com esse cenário de pandemia e isolamento social, o grupo encontrou maneiras adequadas e efetivas de manter o conhecimento vivo, trocar ideias, adquirir informações novas e ainda manter contato, embora de forma remota, com pacientes que necessitam de assistência..."

- “...No front da pandemia, foi possivel sentir os efeitos do aprendizado, pois as sessões científicas e os encontros semanais traziam sempre discussões importantes de artigos atuais e inovadores assim como experiências compartilhadas pelos demais integrantes no grupo, fundamentando de forma conceitual a prática pautada em evidências..."

- “...Coerência metodológica entre as práticas e temas envolvidos, conhecimento quanto ao uso de novas ferramentas de ensino e aprendizagem e a construção do conhecimento compartilhada, se traduzem para mim, como a inovação no ensino urgente pela pandemia..."

- “... O comparecimento nas reuniões da sessão científica, proporcionou-me um maior conhecimento, a partir da interpretação de artigos, os quais apresentaram temas muito pertinentes sobre hipertensão arterial e como os enfermeiros podem colaborar para proporcionar um melhor atendimento a esses pacientes, utilizando terapias complementares, autocuidado..."

- “...Além disso, as Lives marcaram uma transição muito importante para o GEpHAS, no aspecto das discussões de eixos temáticos de foco da linha de pesquisa do grupo serem abertas ao público, a partir do Instagram. Como também é indescritível poder ter aprendido tanto com os convidados de cada Live..."

Fonte: Dados GEpHAS.

Quanto ao "Aprendizado", sabe-se que a pandemia da Covid-19 descortinou para profissionais e educadores mais experientes, uma nova perspectiva de aprendizado, necessitando de adaptações rápidas e até então desconhecidas no universo digital (Carolan, 2020). Ademais, o impacto no aprendizado é de longo alcance e ainda desconhecido, destacando a necessidade iminente de inovação e implementação de estratégias alternativas que garantam a continuidade da educação e avaliação (Dhawan, 2020).

No momento, faz-se possível definir a aprendizagem online como experiências de aprendizagem em ambientes síncronos ou assíncronos usando dispositivos distintos como telefones celulares e laptops, com acesso a Internet, cujo educando pode estar em qualquer lugar para aprender e interagir com instrutores e outros alunos (Singh \& Thurman, 2019). E diante do contexto da aprendizagem, é primordial que o conteúdo abordado não se limite apenas a um ato informativo e que o preceptor promova motivação, confiança e engrandeça a experiência desse processo (Rhin \& Han, 2020). O aprender de forma online permitiu superar as barreiras físicas e temporais do ensino e, com a pandemia, esse aprender torna-se um método educacional eficaz que deve estar vinculado, principalmente, ao diálogo, para que as distâncias entre os integrantes sejam diminuídas (Rhin \& Han, 2020).

Com isso, um estudo de pesquisa de opinião reforça o ganho no aprendizado em ambiente virtual, visto que em uma amostra de 171 acadêmicos de fisioterapia questionados sobre o nível de aprendizado por metodologias ativas durante a pandemia da Covid-19, 135 (79\%) corresponderam que a modalidade de ensino por interação online mostrou-se como efetiva, sendo um meio de ensino ativo e de desenvolvimento do raciocínio crítico (Dosea et al., 2020). Portanto, esses pontos ratificam os relatos dos acadêmicos e pós-graduandos, no sentido de expressarem as vivências de um aprendizado inovador, com enriquecimento científico e motivador para busca de conhecimentos.

E de acordo com os autorrelatos, percebe-se, principalmente, a riqueza do aprendizado durante os últimos 12 meses.

Quando olhamos o Quadro 4, sobre a contribuição para a formação, os autorrelatos se baseiam na significância e compromisso com o conhecimento adquirido seja na graduação ou na pós-graduação.

Visto que, mesmo diante das adversidades impostas pela pandemia provocada pela Covid-19, é necessário garantir o diálogo entre profissionais, docentes e discentes de modo a permitir a produção do conhecimento alinhado os avanços da ciência e a articulação de vários conhecimentos, superando a dicotomia entre a teoria e prática (Pinho, 2017). 
Tanto a produção científica quanto a formação profissional de excelência são fortalecidas pela participação e engajamento dos acadêmicos em grupos de pesquisa. As atividades desempenhadas nesses espaços, como debates e encontros periódicos, promoção de eventos científicos e divulgação de informações relativas à prática baseada em evidências, contribuem ao aprimoramento dos saberes e práticas, tendo em vista as experiências anteriormente relatadas que ratificam o que descreve a literatura científica (Erdmann, 2017).

Neste cenário, a inserção de estudantes de enfermagem de graduação e pós-graduação em grupos de pesquisa, sob a supervisão de docentes-pesquisadores, fomentam a aproximação com a ato de investigar, e consequentemente, o desenvolvimento de raciocínio crítico e reflexivo quanto a prática assistencial, gerência e ensino (Azevedo et al. 2018).

Corrobora com este pensamento, Pinho (2017) ao defender que a participação em grupo de pesquisa, iniciação científica, congressos e publicações em revistas científicas, estimula talentos potenciais nos estudantes, o que permite a socialização profissional, ampliação do conhecimento e a possibilidade de crescimento pessoal.

Ainda para Azevedo et al. (2018), o grupo de pesquisa é considerado um espaço favorável que viabiliza o intercâmbio de conhecimentos, troca de experiências e a discussão de resultados de pesquisas científicas para a incorporação das melhores práticas baseadas em evidências nos serviços de saúde e na prática profissional da enfermagem.

Silva et al. (2018) reforçam que os grupos de pesquisas no âmbito da enfermagem emergem como uma ferramenta multifacetada que permite o fortalecimento de bases científicas, revelada pela aquisição de novos saberes mediada por estudantes, enfermeiros assistenciais e pesquisadores.

Portanto, percebe-se que as atividades desenvolvidas em grupos de pesquisa permitem uma construção de conhecimentos que favorecem uma maior qualificação da formação acadêmica, possibilitando uma reflexão sobre a importância da implementação dessas práticas científicas e a necessidade de uma inserção cada vez maior dos estudantes, para que o cuidado prestado ao paciente se desenvolva da melhor maneira possível (Rossit, 2018). 
Quadro 4. Pontos em destaque para o autorrelato dos membros do grupo de pesquisa quanto a formação e participação no telemonitoramento. Niterói,2021.

\section{FORMAÇÃO}

- “...Outro momento significativo para a minha formação foram as Sessões Científicas. Com os nossos encontros semanais foi possivel ter contato e ouvir relatos de enfermeiras da prática assistencial e a sua atuação diante do cenário da COVID-19. Elas traziam experiências a respeito dos atendimentos prestados, como a enfermagem estava atuando e a associação da COVID-19 com os quadros cardiovasculares apresentados pelos pacientes, permitindo a compreensão da realidade e contribuindo muito para o nosso aprendizado..."

- “..Eu comecei meus estudos com o grupo GEpHAS este ano, 2020, e posso dizer que me trouxe muito conhecimento e sabedoria..."

- “...Diante disso, o grupo tem sido uma parte fundamental do meu processo acadêmico, pois vem me modulando como um aluno melhor uma pessoa melhor, sendo assim, um enfermeiro melhor, pois, antes de tudo somos seres humanos e o enfoque do grupo é nisso, no acolhimento, autocuidado e no olhar para nós mesmos e assim podendo transmitir o melhor cuidado possível. Fique claro, portanto, a importância do grupo para minha formação, em todos os âmbitos, pois além do raciocínio clínico e científico que o grupo propõe através dos encontros, as atividades realizadas são de total importância para o meio acadêmico e para a população..."

- “... a experiência vivenciada nesse periodo remoto no GEPHAS corroboram significativamente para a formação acadêmica de um enfermeiro, mestrando ou doutorando. Isso porque, mesmo em momentos de dificuldade enfrentadas no país como um todo, houve transformações e lapidações de ferramentas e temáticas, de forma a proporcionar melhorias no saber mesmo em tempos de isolamento social causados pela pandemia da COVID-19..."

- “...O grupo contribuiu grandiosamente na minha formação, com certeza termino o ano totalmente diferente de como iniciei, aprendi muito com cada componente e suas falas durante as participações. A dedicação de todos e a sabedoria da líder fizeram a diferença na construção do conhecimento conjunto pela maneira que os debates eram conduzidos e desenvolvidos. Portanto, fazer parte do grupo foi de fato relevante para o meu aprendizado e muito gratificante..."

\section{PARTICIPAÇÃO NO TELEMONITORAMENTO}

- “...A atividade de telemonitoramento é sempre um momento muito especial uma vez que, os pacientes hipertensos, classificados como grupo de risco, demonstraram-se mais preocupados durante a chamada telefônica. Proporcioná-los informação, escuta passiva, educação em saúde, contribuir para resolução de pequenos problemas, contextualizá-los quanto ao momento da saúde no cenário mundial contribuiu muito para o meu aprendizado e conhecimento, promovendo benefícios não e a adesão ao tratamento e medidas protetivas provocam fortes impactos não só na esfera emocional mas principalmente, educacional..."

- “...Como foi importante neste periodo de pandemia estar próximo dos pacientes, orientá-los mediante as instruções da professora Dayse, estabelecendo vínculo e também muito aprendizado. Com essa atividade foi possível ouvir as demandas dos pacientes, estar em contato com a professora e essa com médicos, buscando resolutividade. Pode-se experimentar um verdadeiro trabalho de equipe, com objetivo de acolher, proporcionar uma comunicação terapêutica e a melhor assistência de enfermagem com comunicação telefônica aos pacientes, em um período de muitas dúvidas e medos pela COVID-19..."

- “...Porém a atividade que mais me marcou nesse ano foi o telemonitoramento realizado com os pacientes do ambulatório.Foi meu primeiro contato com esse tipo de atividade e a oportunidade de se construir um vínculo com o paciente e promover saúde de forma remota é de extrema relevância para mim como graduanda e futura profissional..."

- “...Participar do telemonitoramento foi uma experiência ímpar. O desenvolvimento deste projeto nos aproximou mais dos pacientes do ambulatório de hipertensão, a desenvolver a empatia ao próximo e a escuta sensível, além de ajudá-los quanto as dúvidas sobre a doença, o cenário mundial e acompanhá-los durante este ano tão difícil..."

Fonte: Autores.

E por fim, quanto a experiência de participação no telemonitoramento, conclui-se a importância da vivência e do estabelecimento de vínculos.

Pois, a pandemia da COVID-19 trouxe um grande desafio para os profissionais da área da saúde, principalmente no tratamento das doenças crônicas, pela dificuldade no acompanhamento por meio de consultas ou atividades presenciais. Com 
isso, o telemonitoramento é tido como uma boa forma para a assistência a estes indivíduos, por meio da realização de uma monitorização efetiva, principalmente em relação a questões sobre saúde e doença (Caetano, et al, 2020).

Portanto, torna-se notável a importância do telemonitoramento de hipertensos em tempos de pandemia, uma vez que por intermédio desta estratégia é possível implementar ações educativas a fim de ofertar melhor compreensão acerca de cuidados para prevenção da COVID-19 e proporcionar maior aproximação em situações de isolamento, além de contribuir para adesão e manutenção ao tratamento desse indivíduo (Nunes et al., 2020).

Logo, torna-se uma alternativa de atendimento eficaz e de apoio a estes indivíduos que necessitam de acompanhamento a longo prazo. Além disso, surge como opção para aperfeiçoamento dos profissionais de enfermagem, que devem se reinventar e buscar novas maneiras de comunicação e de realização da educação em saúde de maneira competente, atentando-se para o entendimento dos pacientes (Caetano, et al, 2020).

Por fim, pode-se dizer que, de fato, a manutenção e reaproximação do contato do grupo, por meio virtual, proporcionou desde então um olhar enriquecido pela troca de impressões, sentimentos e valorização do aprendizado e conhecimento de todos.

\section{Conclusão}

A experiência compartilhada acerca das atividades científicas referentes à hipertensão arterial no contexto de saúde e da pandemia da Covid-19, foi composta por ações quantitativas direcionadas ao planejamento anual e dos autorrelatos dos membros do grupo de pesquisa.

Observa-se que a pandemia trouxe desafios de toda ordem, seja no campo pessoal ou profissional, imprimindo a urgente busca por nova reorganização aqui em destaque no campo da pesquisa. Essa experiência dos últimos 12(doze) meses demonstrou o quanto se faz importante a dinâmica da continuidade na discussão, participação e contribuição nas múltiplas atividades de um grupo, seja de modo virtual, como possível para o momento.

\section{Referências}

Azevedo, I. C., Silva, R. C. L., Carvalho, D. P. S. R. P., Cruz, G. K. P., Lima, J. V. H., \& Júnior, M. A. F. (2018). Importância do grupo de pesquisa na formação do estudante de enfermagem. Revista de Enfermagem da UFSM. 8 (2): 390-398. https://periodicos.ufsm.br/reufsm/a rticle/view/26003\#: :text=Considera\%C3\%A7\%C3\%B5es\%20finais\%3A\%20o\%20envolvimento\%20nos,de\%20uma\%20pr\%C3\%A1tica\%20pensada\%2Fref lexiva

Barreto, A. C. F. \& Rocha, D. S. (2020). Covid 19 e educação: resistências, desafios e (im)possibilidades. Revista Encantar - Educação, Cultura e Sociedade. 2(10): 01-11. https://revistas.uneb.br/index.php/encantar/article/view/8480/0

Barros, G. M., Filho, J. B. R. M., \& Júnior, A. C. M. (2020). Considerações sobre a relação entre a hipertensão e o prognóstico da COVID-19. J. Health Biol Sci. 8(1):1-3. https://periodicos.unichristus.edu.br/jhbs/article/view/3250

Barroso, W. K. S., Rodrigues, C. S., Bortolotto, L. A., Gomes, M. M., Brandão A. A., Feitosa A. D. M., et al. (2021). Diretrizes Brasileiras de Hipertensão Arterial. Arq Bras Cardiol. 116(3): 516-658. https://abccardiol.org/article/diretrizes-brasileiras-de-hipertensao-arterial-2020/

Brasil. Ministério da Saúde. COVID-19 no Brasil. (2021) https://susanalitico.saude.gov.br/extensions/covid-19_html/covid-19_html.html

Caetano, R., Silva, A. B., Guedes, A. C. C. M., Paiva, C. C. N. D., Ribeiro, G. D. R., Santos, D. L., \& Silva, R. M. D. (2020). Desafios e oportunidades para telessaúde em tempos da pandemia pela COVID-19: uma reflexão sobre os espaços e iniciativas no contexto brasileiro. Cadernos de Saúde Pública. 36(5):e00088920. https://www.scielo.br/scielo.php?script=sci_arttext\&pid=S0102-311X2020000503001

Carolan, C., Davies, C. L., Crookes, P., McGhee, S., \& Roxburgh, M. (2020). COVID 19: Impactos disruptivos e oportunidades transformadoras no ensino de graduação em enfermagem. Enfermeira educação na prática. 46: 102807. https://doi.org/10.1016/j.nepr.2020.102807

Cook, T. M. (2020). The importance of hypertension as a risk factor for severe illness and mortality in COVID-19. Anaesthesia. 75(7): 97677. https://www.ncbi.nlm.nih.gov/pmc/articles/PMC7267452/

Dhawan, S. (2020). Aprendizagem online: uma panaceia na época da crise do COVID-19. Journal of Educational Technology Systems. 49 (1): 5-22. https://doi.org/10.1177/0047239520934018 
Dosea, G. S., Rosário, R. W. S., Silva, E. A, Firmino, L. R., \& Oliveira, A. M. S. (2020). Métodos ativos de aprendizagem no ensino online: A opinião de universitários durante a pandemia de COVID-19. Interfaces Científicas. 10(1):137-148. https://periodicos.set.edu.br/educacao/article/view/9074/4134

Erdmann, A. L., Peiter, C. C. \& Lanzoni, G. M. M. (2017). Brazilian research groups in nursing: comparison of 2006 and 2016 profiles. Revista Gaúcha de Enfermagem. 38(2): e69051. https://www.scielo.br/scielo.php?pid=S1983-14472017000200418\&script=sci_arttext\&tlng=en

Harapan, H., Itoh, N., Yufika, A., Winardi, W., Keam, S., Te, H., et al. (2020). Coronavirus disease 2019 (COVID-19): A literature review. Journal of Infection and Public Health. 13(5): 667-673. https://doi.org/10.1016/j.jiph.2020.03.019

Lei n. 14.040, de agosto de 2020. Estabelece normas educacionais excepcionais a serem adotadas durante o estado de calamidade pública reconhecido pelo

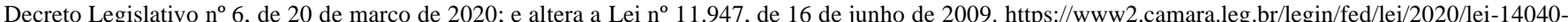
18-agosto-2020-790546-veto-161319-pl.html\#: :text=Estabelece\%20normas\%20e ducacionais\%20excepcionais\%20a,16\%20de\%20junho\%20de\%202009

Nunes, A. S. A., Reis, A. C. F., Pires, A. S., Freitas, B. J. (2020). Uso do telemonitoramento e telenfermagem como estratégia de cuidado ao paciente crônico ambulatorial durante a pandemia covid-19. Atena Editora. 228-240. https://www.finersistemas.com/atenaeditora/index.php/admin/api/artigoPDF/42001

Oliveira, V. H. N. (2021). Desafios para a pesquisa no campo das ciências humanas em tempos de pandemia da COVID-19. Boletim de Conjuntura (BOCA). 5(14):1-11. http://revista.ioles.com.br/boca/index.php/revista/article/view/211/221

Pereira, A. S., et al. (2018). Metodologia da pesquisa científica. UFSM. https://repositorio.ufsm.br/bitstream/han dle/1/15824/Lic_Computacao_MetodologiaPesquisa-Cientifica.pdf?sequence $=1$

Pinho, M. J. (2017). Ciência e ensino: contribuições da iniciação científica na educação superior. Revista da Avaliação da Educação Superior. 22(3): 658675. https://www.scielo.br/scielo.php?pid=S141440772017000300658\&script=sci_abstract\&tlng=pt

Resolução 510 de 07 de abril de 2016.Conselho Nacional de Ética em Pesquisa (CONEP). Dispõe sobre as normas aplicáveis a pesquisas em Ciências Humanas e Sociais cujos procedimentos metodológicos envolvam a utilização de dados diretamente obtidos com os participantes ou de informações identificáveis ou que possam acarretar riscos maiores do que os existentes na vida cotidiana, na forma definida nesta Resolução http://conselho.saude.gov.br/resolucoes/2016/Reso510.pdf

Rhim, H. C. \& Han, H. (2020) Teaching online: foundational concepts of online learning and practical guidelines. Korean Journal of Medical Education. 32(3): 175-183. https://www.kjme.kr/journal/view.php?doi=10.3946/kjme.2020.171

Rodgers, G. P., \& Gibbons, G. H. (2020). Obesity and Hypertension in the time of COVID-19. JAMA. 324 (12): 116372. https://jamanetwork.com/journals/jama/fullarticle/2770253

Rossit, R. A. S., Santos Junior, C. F., Medeiros, N. M. H., Medeiros, L. M. O. P., Regis, C.G. \& Batista, S. H. S. S. (2018). Grupo de pesquisa como espaço de aprendizagem em/sobre educação interprofissional (EIP): narrativas em foco. Interface - Comunicação, Saúde, Educação. 22(2): 15111523.https://www.scielo.br/scielo.php?script=sci_arttext\&pid=S1414-32832018000601511\&lng=pt\&tlng=pt

Salazar, M., Barochiner, J., Espeche, W. \& Ennis, I. (2020). Covid-19, hipertensión y enfermedad cardiovascular. Hipertens Riego Vasc. 37(4): 176180. https://www.ncbi.nlm.nih.gov/pmc/articles/PMC7301092/

Silva, I. R., Leite, J. L, Trevizan, M. A., Silva, T, P., \& Mendes, I. A. C. (2018). Grupos de pesquisa em enfermagem: sistemas complexos para a gestão do conhecimento. Rev Min Enferm. 22:2-8. http://www.reme.org.br/artigo/detalhes/1246\#: :text=Os\%20grupos\%20de\%20pesquisa\%20surg em,de $\% 20$ conhecimento $\% 20$ cient $\%$ C3\%ADfico $\% 20$ na $\% 20$ enfermagem.\&text=Desse $\% 20$ processo $\% 2 \mathrm{C} \% 20$ poder $\% 2 \mathrm{Dse} \% 2 \mathrm{D}, \mathrm{de} \% 20 \mathrm{cunho} \% 20 \mathrm{geral} \% 20 \mathrm{e} \%$ 20 espec\%C3\%ADfico

Singh, V., \& Thurman, A. (2019). How many ways can we define online learning? A systematic literature review of definitions of online learning (19882018). American Journal of Distance Education. 33(4): 289-306. https://www.researchgate.net/publication/336170577_How_Many _Ways_Can_We_Define_Online_Learning_A_Systematic_Literature_Review_of_Definitions_of_Online_Learning_1988-2018

Souza, E. P. (2020). Educação em tempos de pandemia: desafios e possibilidades. Caderno de Ciências Sociais Aplicadas.7(30): 01-09. https://periodicos2.uesb.br/index.php/ccsa/article/view/7127/5030

Teich, V. D., Klajner, S., Almeida, F. A. S.de, Dantas, A. C. B., Laselva, C. R.,Torritesi, M. G., et. al. (2020). Características epidemiológicas e clínicas dos pacientes com COVID-19 no Brasil. Einstein. 18(1):1-7. https://journal.einstein.br/wp-content/uploads/articles_xml/2317-6385-eins-18eAO6022/2317-6385-eins-18-eAO6022-pt.pdf?x56956 